\title{
Le radon 222, agent potentiellement nocif dans certaines mines non uranifères
}

\author{
H. WEISSBUCH, E. BOTEZATU et M. GRĂDINARU (*) \\ (Manuscrit reçu le 11 octobre 1978)
}

\begin{abstract}
RÉSUMÉ
Dans certaines mines souterraines, les travailleurs sont exposés à des concentrations élevées de radon dans l'atmosphère.

Pour estimer le risque potentiellement cancérigène pour les mineurs, on a choisi sept mines sans uranium. Dans chaque mine on a determiné les concentrations de ${ }^{222} \mathrm{Rn}$ et de ses descendants à faible période, ainsi que celles des radioéléments naturels $\left(\mathrm{U},{ }^{232} \mathrm{Th},{ }^{40} \mathrm{~K}\right)$ dans les roches et les effluents de la mine. Ce travail présente les résultats concernant les concentrations de ${ }^{222} \mathrm{Rn}$ dans l'atmosphère des mines étudiées. A l'exception d'une seule mine, les valeurs moyennes des concentrations mesurées dans les mines dépassent la concentration maximale admissible pour les travailleurs directement affectés aux travaux sous rayonnements ionisants (norme CIPR) et a fortiori la CMA pour la population. La présence de ${ }^{222} \mathrm{Rn}$ à des concentrations élevées constitue un indicateur du risque potentiellement cancérigène pour les mineurs exposés sans nous permettre l'évaluation quantitative de ce risque.
\end{abstract}

\begin{abstract}
Workers in certain underground mines are exposed to high levels of radon present in the atmosphere of the mines.

To estimate the potential carcinogen risk for miners, 7 non-uranium mines were studied. Concentrations of ${ }^{222} \mathrm{Rn}$ and its short-lived-daughter products in air, and of natural radionuclides $\left(\mathrm{U},{ }^{232} \mathrm{Th},{ }^{40} \mathrm{~K}\right)$ were determined in rocks and mine waters. The results of ${ }^{222} \mathrm{Rn}$ measurements carried out in these mines are presented. But for one mine, the mean value of ${ }^{222} \mathrm{Rn}$ concentrations in the mine atmospheres exceeded both the maximum permissible value for members of the public, and the maximum permissible concentration (MPC) in air for occupational exposure (ICRP value). The presence of high ${ }^{222} \mathrm{Rn}$ concentrations represents an indicator of the miners' potentiel carcinogen risk, without permitting a quantitative estimation of this risk.
\end{abstract}

(*) Institutul de igienă si sănătate publică, Str. Dr. Victor Babes nr 14, IASI, Roumanie. 


\section{INTRODUCTION}

Les premières observations sur une surmortalité par des maladies pulmonaires chez certaines catégories de mineurs datent du siècle dernier. Ainsi, on connaît la mortalité anormalement élevée due à une affection appelée "Schneebergerkrankheit » qui frappait les travailleurs des mines souterraines de Schneeberg (Saxonie) et de Joachimstahl (Tchécoslovaquie) [4]. Mais ce n'est qu'en 1876, en Saxe, et en 1926, en Tchécoslovaquie, que cette maladie a été identifiée comme étant un cancer du poumon. En ce qui concerne l'agent étiologique de ce type spécial de cancer professionnel, il s'agit du gaz radioactif ${ }^{222} \mathrm{Rn}$ et de ses descendants à faible période $\mathrm{RaA}$ et $\mathrm{RaC}[2]$. Le radon en tant que nuisance professionnelle n'est pas spécifique aux mines d'uranium. On a également signalé la surmortalité par cancer du poumon chez les mineurs de spathfluor au Canada, de fer en Grande-Bretagne $[1,3]$ de tungstène, de spathfluor et de lithium en Tchécoslovaquie [7], etc. Le personnel du fond de toutes ces mines avait été professionnellement exposé à de fortes doses de radon [7]. D'après les investigations effectuées par un groupe de chercheurs du Trust Metale Rare de Roumanie dans certaines mines non uranifères, le ${ }^{222} \mathrm{Rn}$ et ses descendants se trouvaient en fortes concentrations.

Notre étude avait pour but de déterminer les concentrations de ${ }^{222} \mathrm{Rn}$ et de ses descendants dans une zone minière et d'estimer le risque cancérigène pour les mineurs de la zone étudiée.

\section{MÉTHODES}

On a choisi pour cette recherche sept mines appartenant au bassin minier du nord de la Moldavie, d'où l'on extrait principalement $\mathrm{Cu}, \mathrm{Mn}, \mathrm{Zn}, \mathrm{Pb}$, $\mathrm{Ba}, \mathrm{Fe}$ et $\mathrm{S}$. Les principaux critères de sélection ont été la composition géologique des minerais et le nombre de travailleurs souterrains. Dans chaque mine, on a effectué des mesures dans plusieurs abattages (pendant l'accomplissement de différentes opérations), dans des niches abandonnées (où ${ }^{222} \mathrm{Rn}$ s'accumule de façon préférentielle), ainsi que dans des galeries d'accès, ceci à différentes profondeurs.

Environ 800 échantillons d'air ont été prélevés en quatre étapes (avril 1974juillet 1975). Dans deux mines, on a mesuré les concentrations de ${ }^{222} \mathrm{Rn}$ en dynamique afin d'estimer ses variations sous l'influence de plusieurs facteurs comme l'aérage, les conditions météorologiques, la nature des opérations effectuées.

L'air chargé de radon était aspiré, à travers un filtre, dans un ballon standard à scintillations préalablement vidé. On mesurait ensuire, à l'aide d'un appareil portatif, l'activité alpha du ${ }^{222} \mathrm{Rn}$ en équilibre avec ses descendants de faible période [6]. La sensibilité de la méthode est de $2 \mathrm{pCi} / \mathrm{l}$ d'air, c'est-à-dire $1 / 15^{e}$ de la CMA professionnelle.

\section{RÉSULTATS ET DISCUSSIONS}

Le ${ }^{222} \mathrm{Rn}$ est un radionucléide gazeux qui, en se désintégrant, donne naissance à d'autres éléments radioactifs, dont les quatre premiers sont des isotopes à faible période de certains métaux lourds : $\mathrm{Po}, \mathrm{Pb}, \mathrm{Bi}$. Ces isotopes 
se fixent sur les aérosols de l'atmosphère. Pénétrant par inhalation dans l'arbre bronchique, les deux radionucléides émetteurs alpha peuvent s'y fixer et soumettre l'épithélium respiratoire à une irradiation à TLE élevé, créant un certain risque cancérigène [7].

Nos résultats montrent que, dans certains endroits, les mineurs sont exposés à de fortes concentrations de ${ }^{222} \mathrm{Rn}$ (tableau).

TABLEAU

CONCENTRATIONS MOYENNES DE ${ }^{22}$ Rn DANS CERTAINES MINES NON URANIFÈRES

\begin{tabular}{|c|c|c|c|c|c|}
\hline \multirow[b]{2}{*}{ Mine } & \multicolumn{3}{|c|}{$\begin{array}{c}\text { Concentrations } \\
\text { de }{ }^{222} \mathrm{Rn}(\mathrm{pCi} / 1 \text { air })\end{array}$} & \multicolumn{2}{|c|}{$\begin{array}{l}\text { Rapport entre concentrations } \\
\qquad \text { de }^{222} \mathrm{Rn} \text { et }\end{array}$} \\
\hline & Moyenne & Maximale & Minimale & $\begin{array}{c}\text { CMA } \\
\text { professionnelle } \\
(40 \mathrm{~h}) \\
\text { (norme CIPR) }\end{array}$ & $\begin{array}{c}\text { CMA } \\
\text { pour } \\
\text { un individu } \\
\text { du public } \\
(168 \mathrm{~h})\end{array}$ \\
\hline A $\ldots \ldots \ldots$ & 67 & 130 & 28 & 2,2 & 6,7 \\
\hline${ }_{B}\left\{B^{1} \ldots\right.$ & 48 & 127 & 6 & 1,6 & 4,8 \\
\hline $\mathrm{B}\left\{\mathrm{B}^{2} \ldots\right.$ & 70 & 267 & 13 & 2,3 & 7,0 \\
\hline C........ & 46 & 185 & 7 & 1,5 & 4,6 \\
\hline $\mathrm{D} \ldots \ldots \ldots$ & 129 & 160 & 41 & 4,0 & 12,9 \\
\hline D........ & 1278 & 1866 & 963 & 42 & 127,8 \\
\hline $\mathrm{E} \ldots \ldots \ldots$ & - & 374 & 4,5 & - & - \\
\hline$F \ldots \ldots \ldots$ & 74 & 111 & 14 & 2,4 & 7,4 \\
\hline$\left(G^{1} \ldots\right.$ & 33 & 56 & 963 & 1,0 & 3,3 \\
\hline$G\left\{G^{2} \ldots\right.$ & 13,1 & 30,2 & 3,5 & 0,4 & 1,3 \\
\hline
\end{tabular}

A l'exeption de la valeur moyenne des concentrations, mesurée dans la mine $\mathrm{G}$, les valeurs moyennes des concentrations des autres mines dépassent tant la valeur limite acceptée pour une personne faisant partie de la population que la CMA professionnelle. Dans les mines $A$ et $B_{2}, 70$ p. cent des valeurs dépassent la CMA professionnelle, tandis que dans la mine $B_{1}$, ce n'est que 36 p. cent des valeurs qui dépassent la CMA.

Les concentrations élevées de radon dans l'atmosphère des mines $\mathrm{A}$ et $\mathrm{B}$ ont pu être mises en corrélation avec les doses gamma externes produites par les radioéléments naturels de la roche, doses qui dépassent de 2 à 11 fois la composante tellurique du fond naturel d'irradiation.

Dans la mine D, on a trouvé une situation toute spéciale, car la CMA professionnelle est dépassée en tous les points de mesure, trois d'entre eux ayant des valeurs particulièrement élevées.

Les valeurs élevées des concentrations de radon 222 trouvées dans la mine D ne peuvent être expliquées, dans l'état actuel de nos recherches, ni par la présence des radioéléments naturels dans les roches ni par teneur en radon des eaux d'infiltration. 
Les fortes concentrations de ${ }^{222} \mathrm{Rn}$ dans l'air des mines indiquent l'existence d'un risque potentiellement cancérigène pour les personnes exposées [2]. La valeur réelle du risque ne peut être mise en corrélation directe avec les concentrations en radon, car l'agent étiologique de ce type spécial de cancer bronchique est représenté par les radionucléides émetteurs alpha à faible période : ${ }^{218} \mathrm{Po}(\mathrm{RaA})$ et ${ }^{214} \mathrm{Po}\left(\mathrm{RaC}^{\prime}\right)$, pour lesquels le radon ne représente que la source génératrice [8].

L'aérage, les concentrations d'aérosols et de poussières, les conditions météorologiques, etc. font varier les rapports entre la concentration du radon et de ses descendants. Il est donc nécessaire de déterminer directement les concentrations des descendants du radon dans l'air des mines [5, 7].

L'objet des recherches que nous poursuivons est l'évaluation des doses reçues par les mineurs, en mesurant directement les concentrations des descendants du radon. On tâche aussi de dépister une surmortalité par le cancer bronchique chez les mineurs exposés à de fortes concentrations de radon, comparaison avec l'incidence de cancer bronchique chez la population masculine dans la zone minière étudiée.

\section{CONCLUSIONS}

1. On a trouvé des concentrations de radon 222 qui dépassent la CMA professionnelle en de nombreux postes de travail des mines étudiées.

2. La présence du radon 222 aux concentrations élevées constitue un indicateur du risque potentiellement cancérigène pour les mineurs exposés, sans nous permettre l'évaluation quantitative de ce risque.

\section{BIBLIOGRAPHIE}

[1] Boyd J. T., Doll R., Faulds J. S. Cancer of the lung in iron ore (haematite) miners. Brit. J. Ind. Med., 1970, 27, 97-105.

[2] De Villiers A. J., Windish J. P. Lung cancer in a fluorspar mining community-radiation dust and mortality experience. Brit. J. Ind. Med., 1964, 21, 94-103.

[3] Duggan M. J., Sollleux P. J., Strong J. C. The exposure of United Kingdom miners to radon. Brit. J. Ind. Med., 1970, 27, 106-109.

[4] HoRACEK J. Der Joachimsthaler Lungenkrebs nach dem zweiten Weltkrieg. Krebsforschung, 1969, 72, 52-56.

[5] JACOBI W. Problems concerning the recommendation of a maximum permissible inhalation intake of short-lived radon daughters. Proceedings of the Second IRPA European Congress on Radiation Protection. Budapest, Akademiai Kiado, 1975, 109-121.

[6] Pradel J., Billard F. Le thoron et les risques associés dans la manipulation des composés du thorium. Rapport CEA-1165, 1959, 6-7.

[7] Rayonnements ionisants: niveaux et effects. Rapport du Comité scientifique des Nations Unies pour l'étude des effets des rayonnements ionisants à l'Assemblée générale et annexes. New York, Nations Unies, 1972, vol. 2, 445-447.

[8] The Effects on populations of exposure to low levels of ionizing radiation. Report of the Advisory Committee on the Biological Effects of Ionizing Radiations (BEIR). Washington, National Academy of Sciences, 1974, 145-156. 